May 1993

DOE-ER 40757-012

CPP-93-12

LTP-038-UPR

\title{
Induced charge of neutrinos in a medium
}

\author{
José F. Nieves \\ Physics Department, University of Puerto Rico, Rio Piedras, PR 00931 \\ Palash B. Pal \\ Center for Particle Physics, University of Texas, Austin, TX 78712, USA
}

\begin{abstract}
Neutrinos can polarize a medium due to their weak interaction. This can manifest itself as an effective induced charge of the neutrino. We show how it is related to the Debye screening length in a plasma, first using the results of the 1-loop calculation of the neutrino electromagnetic vertex and then in general to all orders in $e$ and to leading order in the Fermi coupling. We also discuss how the results are modified if the neutrinos have mass - either Dirac or Majorana type.
\end{abstract}

PACS numbers: $14.60 . \mathrm{G}$ 


\section{Introduction}

The properties of neutrinos that propagate through a medium have been the subject of great interest in the recent literature. This has been motivated by the attractive suggestion that the solar neutrino problem |1] can be solved by the resonant oscillation mechanism [2], which hinges on the characteristics of the neutrino propagation in a background medium [3]. Inspired by its potentially important effects, the neutrino interactions in a material environment have also been studied in some detail [4, 5, 6, 7, 8, 9, 10, 11, 12]. Of primary interest along these lines is the study of the electromagnetic interactions of neutrinos in a medium. A classic problem in this field is the decay of a plasmon into $\nu \bar{\nu}$ pairs [13], which has received considerable attention recently [8, 11, 14]. It has also been pointed out that the rate of decay of a massive neutrino into a lighter neutrino and a photon increases tremendously [9, 10] in matter, as a consequence of the fact that the GIM mechanism is not operative in a medium with electrons but no muons or taons.

The study of the electromagnetic interactions of neutrinos in a medium, as well as the results and conclusions mentioned above, are based on the 1-loop calculation of the effective electromagnetic vertex of the neutrino, which has been performed to the leading order in the Fermi constant [8] using the methods of "Quantum Statistical Field Theory" "T. The implications of this calculation have been only partially explored. In this article, we show that the results of Ref. [8] imply that the neutrino acquires a small effective charge in a medium. This point was realized by Oraevsky and Semikoz [6] using methods of plasma physics before the calculation of the $\nu \nu \gamma$ vertex was performed. On the other hand, our method is entirely different, hopefully easier to follow for a particle physicist, and it brings out some interesting points which are not easy to see in the method used by Oraevsky and Semikoz [6]. In addition, we also calculate the induced charge for neutrinos which are massive, distinguishing between the cases of Dirac and Majorana masses.

The plan of the paper is as follows. In Section 2 we establish notation and the definition of the induced charge that is used in the rest of the paper, and show why the neutrino can acquire an induced charge in the medium but not in the vacuum. Then, using field-theoretic methods, we will show that the neutrino electromagnetic vertex is related to the photon self-energy in the medium and, in particular, the neutrino induced charge is related to the Debye screening length in a plasma. This relation is derived to 1-loop order in Section 3, and then in Section 4 we show that it is valid to all orders in $e$ (and to first order in the Fermi coupling). In Section 5 we use this relation to find an expression for the induced charge of the neutrino, and in Section 6 we show that the magnitude of the induced charge depends on whether the neutrinos are massless or massive and, in the latter case, on whether the mass is Dirac or Majorana type. Finally, using the results of Ref. [8], we will estimate the induced charge in some particular backgrounds.

\section{Electromagnetic vertex of the neutrino and the definition of the in- duced charge}

We begin by understanding the reason why the neutrino can acquire an induced charge in the medium but not in the vacuum. The off-shell electromagnetic vertex function $\Gamma_{\lambda}$ is defined in such a way that, for on-shell neutrinos, the $\nu \nu \gamma$ amplitude is given by

$$
M=-i \bar{u}\left(k^{\prime}\right) \Gamma_{\lambda} u(k) A^{\lambda}(q),
$$

where

$$
q \equiv k-k^{\prime}
$$

\footnotetext{
${ }^{1}$ More often, this is called the "Finite Temperature Field Theory", but the name is misleading because the methods are also applicable at zero temperature with a finite density of particles in the background.
} 
is the momentum carried by the photon. In general, $\Gamma_{\lambda}$ depends on $k$ and $k^{\prime}$ or, equivalently, on $k$ and $q$. For neutrinos in a medium $\Gamma_{\lambda}$ depends also on the parameters characterizing the medium. For homogeneous and isotropic media, to which we will restrict ourselves, there is only one such parameter, viz. the velocity 4 -vector of the background medium $v^{\mu}$.

There are two important consequences of the fact that the external (neutrino) lines in the Feynman diagram for the $\nu \nu \gamma$ amplitude are neutral. Firstly, $\Gamma_{\lambda}$ satisfies

$$
q_{\lambda} \Gamma^{\lambda}=0
$$

for all values of $q$. It is important to realize that for neutrinos Eq. (2.3) holds for arbitrary values of $q$, and not just when $k$ and $k^{\prime}$ are on shell. If the fermion lines in the diagram were to correspond to a charged particle (e.g., the electron), then the analogous relation in that case involves terms in the right-hand side involving the inverse propagators corresponding to the external fermions. In that case Eq. (2.3) does not hold for arbitratry values of $q$, but only when both $k$ and $k^{\prime}$ are on shell.

The other important consequence of the fact that the external lines are neutral is that $\Gamma_{\lambda}$ is well defined in the limit $q^{\mu} \rightarrow 0$. The reason is that the photon vertex must be connected to a pair of internal lines of the diagram. If one of these lines is assigned the loop momentum $p$ which is integrated over, the other line will carry a momentum $p \pm q$. The propagator of this second line will involve the factor

$$
\frac{1}{(p \pm q)^{2}-m^{2}}=\frac{1}{q^{2} \pm 2 p \cdot q+\left(p^{2}-m^{2}\right)}
$$

where $m$ is the mass of the internal line. However, since $p^{2} \neq m^{2}$ for any internal line, no singularity is produced for $q^{\mu} \rightarrow 0$.

From the two properties of $\Gamma_{\lambda}$ just discussed, we obtain

$$
\Gamma_{\lambda}\left(q^{\mu}=0\right)=0
$$

which implies that the particle associated with the external line does not acquire a charge in any order of perturbation theory. To see this explicitly, we will consider the matrix element of the charge operator between two neutrino states with momenta:

$$
k^{\lambda}=(E, \vec{k}), \quad k^{\prime \lambda}=\left(E, \vec{k}^{\prime}\right) .
$$

We use states with the same energy, because then

$$
q \equiv k-k^{\prime}=(0, \vec{q})
$$

with $\vec{q}=\vec{k}-\vec{k}^{\prime}$, which corresponds to the static limit. Denoting by $\rho(x)$ the charge density operator, the effective charge is defined by the equation

$$
\begin{aligned}
e_{\mathrm{eff}}\left\langle k^{\prime} \mid k\right\rangle & =\int d^{3} x\left\langle k^{\prime}|\rho(x)| k\right\rangle \\
& =(2 \pi)^{3} \delta^{(3)}(\vec{q})\left\langle k^{\prime}|\rho(0)| k\right\rangle
\end{aligned}
$$

On the other hand,

$$
\left\langle k^{\prime}|\rho(0)| k\right\rangle=\bar{u}\left(k^{\prime}\right) \Gamma_{0}(0, \vec{q}) u(k),
$$

where the notation $\Gamma_{\lambda}\left(q^{0}, \vec{q}\right)$ has been used to indicate explicitly that we are considering the dependence of $\Gamma$ separately on the frequency and wavelength of the photon. Thus we obtain

$$
e_{\mathrm{eff}}=\frac{1}{2 E} \bar{u}(k) \Gamma_{0}(0, \vec{q} \rightarrow 0) u(k)
$$


which is the basic equation to interpret our results, but it can be cast in an elegant way. Introducing the spinor projection matrix

$$
S(k) \equiv u(k) \otimes \bar{u}(k)=\frac{1}{2}\left(1+\lambda \gamma_{5}\right) \not k,
$$

where the last step is valid for massless Weyl spinors with $\lambda= \pm 1$ being the helicity, we can rewrite Eq. (2.10) as

$$
\begin{aligned}
e_{\mathrm{eff}} & =\frac{1}{2 E} \operatorname{tr}\left[\Gamma_{0}(0, \vec{q} \rightarrow 0) S(k)\right], \\
& =\frac{1}{4 E} \operatorname{tr}\left[\Gamma_{0}(0, \vec{q} \rightarrow 0)\left(1+\lambda \gamma_{5}\right) \not k\right],
\end{aligned}
$$

where again the second step is valid for massless Weyl spinors.

Since $\Gamma_{\lambda}$ has a well defined limit as $q^{0} \rightarrow 0$, we can make a Taylor expansion around $q^{0}=0$ :

$$
\begin{aligned}
\Gamma_{0} & =G_{0}+q^{0} G_{1}+O\left(q^{0^{2}}\right) \\
\vec{\Gamma} & =\vec{H}_{0}+q^{0} \vec{H}_{1}+O\left(q^{0^{2}}\right),
\end{aligned}
$$

where all the coefficients are independent of $q^{0}$. Then from Eq. (2.3) it is easy to deduce that, in the limit $q^{0} \rightarrow 0$

$$
\begin{aligned}
\Gamma_{0} & =\vec{q} \cdot \vec{H}_{1}+O\left(q^{0}\right) \\
\vec{\Gamma} & =q^{0} \vec{H}_{1}+O\left(q^{0^{2}}\right) .
\end{aligned}
$$

Since $\vec{H}_{1}$ has a well defined limit as $\vec{q} \rightarrow 0$, it follows that $\Gamma_{0}=0$ in this limit, and from Eq. (2.13) we see that $e^{(\nu)}$ is zero in the vacuum.

In the medium, Eq. (2.3) continues to hold for any value of $q$, and the relations in Eq. (2.15) are also valid, but $\vec{H}_{1}$ no longer has a well defined limit as $\vec{q} \rightarrow 0$. There are several ways to understand why this is so. One of them is to notice that in the case of the medium, some of the internal lines to which the photon is attached are on shell because they correspond to particles that are in the background. Thus, the singularities that are avoided in the case of the vacuum because the photon is attached only to internal off-shell lines, reappear here. Therefore, nothing prevents $\vec{H}_{1}$ to develop a singularity as $\vec{q} \rightarrow 0$ of the form

$$
\vec{H}_{1}=(\text { constant }) \cdot \frac{\vec{q}}{\vec{q}^{2}}
$$

In such a case, the constant appearing in this equation is the value of $\Gamma_{0}$ in the limit $q^{0}=0, \vec{q} \rightarrow 0$. Thus, from the definition of the effective charge in Eq. (2.13), it follows that $e_{\text {eff }}^{(\nu)}$ is non-vanishing.

In what follows, we will show that this is exactly what happens, first explicitly by using the 1-loop calculation of the neutrino electromagnetic vertex and then by a general field-theoretic argument, which extends the 1-loop result to all orders in $e$. This will be done by showing that $\Gamma_{\lambda}$ is related to the photon self-energy $\pi_{\lambda \rho}(q)$. Further, in the limit that we are considering, $\pi_{\lambda \rho}\left(q^{0}=0, \vec{q} \rightarrow 0\right)$ is related to the Debye screening length, which allows us to establish the relation between the latter quantity and the neutrino induced charge.

\section{One-loop result}

We are interested in the regime where the neutrino momenta are small compared to the masses of the $W$ and $Z$ bosons. Therefore, we can neglect the momentum dependence in the $W$ and $Z$ propagators, 
which is justified if we are performing a calculation to the leading order in the Fermi constant $G_{F}$. In this approximation, the diagrams contributing to the electromagnetic vertex then appear at the 1-loop level, and are shown in Fig. 1. Since the momentum dependence of the weak gauge bosons are neglected, these two diagrams can be represented by the single diagram of Fig. 2 with a four-fermion vertex. Let us denote the 4 -fermion interaction by

$$
\mathcal{L}_{\text {int }}^{(\text {weak })}=-\sqrt{2} G_{F}\left[\bar{\nu} \gamma^{\rho} L \nu\right]\left[\bar{f} \gamma_{\rho}\left(\mathcal{A}+\mathcal{B} \gamma_{5}\right) f\right]
$$

where $L=\frac{1}{2}\left(1-\gamma_{5}\right)$ is the projection operator for left chirality, and $f$ stands for the electron field. We can then write the amplitude of Fig. 2 as

$$
\begin{aligned}
-i \Gamma_{\lambda}=(-i e)\left(-i G_{F} \sqrt{ } 2\right)(-1) \gamma^{\rho} L \times \\
\\
\quad \int \frac{d^{4} p}{(2 \pi)^{4}} \operatorname{tr}\left[\gamma_{\lambda} i S_{F}(p) \gamma_{\rho}\left(\mathcal{A}+\mathcal{B} \gamma_{5}\right) i S_{F}(p-q)\right],
\end{aligned}
$$

where $i S_{F}(p)$ denotes the propagator of the background particles with momentum $p$, and $e$ is the charge of the electron. In complicated systems, this propagator may be complicated, and the integration over the momentum $p$ may have unusual measure as well, but we do not need these explicitly for what follows.

Now the interesting observation is that the contribution from $\mathcal{A}$ in Eq. (3.2) is intimately related to the vacuum polarization of the photon which, at 1-loop, arises from the diagram in Fig. 3 and is given by

$$
i \pi_{\lambda \rho}(q)=(-i e)^{2}(-1) \int \frac{d^{4} p}{(2 \pi)^{4}} \operatorname{tr}\left[\gamma_{\lambda} i S_{F}(p) \gamma_{\rho} i S_{F}(p-q)\right] .
$$

Therefore, Eq. (3.2) can be written in the form

$$
\Gamma_{\lambda}=-\frac{G_{F}}{\sqrt{2} e} \gamma^{\rho}\left(1-\gamma^{5}\right)\left(\mathcal{A} \pi_{\lambda \rho}+\mathcal{B} \pi_{\lambda \rho}^{5}\right)
$$

where we have defined

$$
i \pi_{\lambda \rho}^{5}=(-i e)^{2}(-1) \int \frac{d^{4} p}{(2 \pi)^{4}} \operatorname{tr}\left[\gamma_{\lambda} i S_{F}(p) \gamma_{\rho} \gamma_{5} i S_{F}(p-q)\right]
$$

The term proportional to $\pi_{\lambda \rho}^{5}$ does not contribute to $\Gamma_{0}$. The reason is that the trace contains a factor of $\gamma_{5}$, and therefore can be non-zero only if there are at least four other $\gamma$-matrices present. Since the terms in a fermion propagator have at most one $\gamma$-matrix, the trace involves $\operatorname{tr}\left(\gamma_{5} \gamma_{\lambda} \gamma_{\rho} \gamma_{\alpha} \gamma_{\beta}\right)=4 i \epsilon_{\lambda \rho \alpha \beta}$. After the $p$ integration this can yield only a term proportional to $\epsilon_{\lambda \rho \alpha \beta} q^{\alpha} v^{\beta}$, which does not contribute to the zeroth component of $\Gamma_{\lambda}$.

\section{Generalization to higher orders}

The above result, based on the 1-loop calculation of the photon self-energy and the neutrino vertex function, can be generalized as follows. The photon self-energy is defined in general by

$$
\begin{aligned}
i \pi_{\lambda \rho} & =(-i e)^{2} \int d^{4} x e^{i q \cdot x}\left\langle T U^{(\mathrm{em})} j_{\lambda}(x) j_{\rho}(0)\right\rangle, \\
& =(-i e)^{2} \int d^{4} x e^{-i q \cdot x}\left\langle T U^{(\mathrm{em})} j_{\lambda}(0) j_{\rho}(x)\right\rangle,
\end{aligned}
$$

where $j_{\lambda}$ is the electron current density

$$
j_{\lambda}=\bar{f} \gamma_{\lambda} f
$$


and

$$
U^{(\mathrm{em})}=\exp \left(i \int d^{4} z \mathcal{L}_{\text {int }}^{(\mathrm{em})}\right)
$$

with

$$
\mathcal{L}_{\text {int }}^{(\mathrm{em})}=-e j_{\lambda} A^{\lambda}
$$

On the other hand, the neutrino vertex function of Eq. (2.1) is defined by

$$
\Gamma_{\lambda}\left(k, k^{\prime}\right)=e \int d^{4} x d^{4} y e^{-i k \cdot x} e^{i k^{\prime} \cdot y}\left\langle T \exp \left(i \int d^{4} z \mathcal{L}_{\text {int }}^{(\text {total })}\right) \nu(y) j_{\lambda}(0) \bar{\nu}(x)\right\rangle_{a},
$$

where

$$
\mathcal{L}_{\text {int }}^{\text {(total) }}=\mathcal{L}_{\text {int }}^{(\text {weak })}+\mathcal{L}_{\text {int }}^{(\text {em })}
$$

The subscript $a$ in Eq. (4.5) is used to indicate that $\Gamma_{\lambda}$ is obtained from the above formula by amputating the propagators corresponding to the external neutrino lines. It is convenient to rewrite $\mathcal{L}_{\text {int }}^{(\text {weak) }}$, given in Eq. (3.1), in the form

$$
\mathcal{L}_{\text {int }}^{\text {(weak) }}=-\sqrt{2} G_{F} \bar{\nu}_{L} \gamma_{\lambda} \nu_{L}\left[\mathcal{A} j_{\lambda}+\mathcal{B} j_{\lambda}^{5}\right]
$$

where

$$
j_{\lambda}^{5} \equiv \bar{f} \gamma_{\lambda} \gamma^{5} f
$$

To first order in $G_{F}$,

$$
\Gamma_{\lambda}=e \int d^{4} x d^{4} y d^{4} z e^{-i k \cdot x} e^{i k^{\prime} \cdot y}\left\langle T U^{(\mathrm{em})} \nu(y) j_{\lambda}(0) \bar{\nu}(x) i \mathcal{L}_{\text {int }}^{\text {(weak) }}(z)\right\rangle_{a}
$$

which, using Eq. (4.7) and amputating the external neutrino lines, reduces to

$$
\begin{aligned}
\Gamma_{\lambda}= & -i e G_{F} \sqrt{2} \mathcal{A} \gamma^{\rho} L \times \\
& \left(\int d^{4} z e^{-i q \cdot z}\left\langle T U^{(\mathrm{em})} j_{\lambda}(0) j_{\rho}(z)\right\rangle+\int d^{4} z e^{-i q \cdot z}\left\langle T U^{(\mathrm{em})} j_{\lambda}(0) j_{\rho}^{5}(z)\right\rangle\right) .
\end{aligned}
$$

Defining

$$
\begin{aligned}
i \pi_{\lambda \rho}^{5} & =(-i e)^{2} \int d^{4} x e^{i q \cdot x}\left\langle T U^{(\mathrm{em})} j_{\lambda}(x) j_{\rho}^{5}(0)\right\rangle \\
& =(-i e)^{2} \int d^{4} x e^{-i q \cdot x}\left\langle T U^{(\mathrm{em})} j_{\lambda}(0) j_{\rho}^{5}(x)\right\rangle
\end{aligned}
$$

we finally obtain the relation

$$
\Gamma_{\lambda}=-\frac{G_{F}}{\sqrt{2} e} \gamma^{\rho}\left(1-\gamma^{5}\right)\left(\mathcal{A} \pi_{\lambda \rho}+\mathcal{B} \pi_{\lambda \rho}^{5}\right)
$$

This expression is the same as Eq. (3.4), except that now it is clear that it is valid in all orders of the electromagnetic interactions. Since $\pi_{\lambda \rho}^{5}$ is a pseudotensor that depends only on $q$ and $v$, it must be proportional to $\epsilon_{\lambda \rho \alpha \beta} q^{\alpha} v^{\beta}$, and therefore it does not contribute to $\Gamma_{0}$ in the rest frame of the medium. If one includes the effects of strong interaction as well, the various occurences of $\mathcal{L}_{\text {int }}^{(\mathrm{em})}$ should be replaced by $\mathcal{L}_{\text {int }}^{(\mathrm{em})}+\mathcal{L}_{\text {int }}^{\text {(strong) }}$, but this would not change Eq. (4.12). 


\section{Expression for the induced charge}

The relation between the induced charge and the Debye screening length is obtained as follows. As already argued, the zeroth-component of $\Gamma_{\lambda}$ is given by

$$
\Gamma_{0}(0, \vec{q} \rightarrow 0)=-\frac{G_{F} \mathcal{A}}{\sqrt{2} e} \gamma^{\rho}\left[1-\gamma^{5}\right] \pi_{0 \rho}(0, \vec{q} \rightarrow 0) .
$$

The most general form of $\pi_{\lambda \rho}$ is [15]

$$
\pi_{\lambda \rho}=\pi_{T} R_{\lambda \rho}+\pi_{L} Q_{\lambda \rho}+\pi_{P} P_{\lambda \rho}
$$

where

$$
\begin{aligned}
R_{\lambda \rho} & \equiv \tilde{g}_{\lambda \rho}-Q_{\lambda \rho}, \\
Q_{\lambda \rho} & \equiv \frac{\tilde{v}_{\lambda} \tilde{v}_{\rho}}{\tilde{v}^{2}}, \\
P_{\lambda \rho} & \equiv \frac{i}{\mathcal{Q}} \epsilon_{\lambda \rho \alpha \beta} q^{\alpha} v^{\beta},
\end{aligned}
$$

with

$$
\begin{aligned}
\tilde{g}_{\lambda \rho} & \equiv g_{\lambda \rho}-\frac{q_{\lambda} q_{\rho}}{q^{2}} \\
\tilde{v}_{\lambda} & \equiv \tilde{g}_{\lambda \rho} v^{\rho} \\
\mathcal{Q} & \equiv \sqrt{(q \cdot v)^{2}-q^{2}}
\end{aligned}
$$

In the rest frame of the medium, with $v^{\mu}=(1,0,0,0)$, the above definitions give

$$
\begin{aligned}
Q_{\lambda \rho}(0, \vec{q}) & =v_{\lambda} v_{\rho}, \\
R_{00}(0, \vec{q})=R_{i 0}(0, \vec{q})=R_{0 i}(0, \vec{q}) & =0, \\
P_{00}(0, \vec{q})=P_{i 0}(0, \vec{q})=P_{0 i}(0, \vec{q}) & =0,
\end{aligned}
$$

where we have indicated explicitly the fact that we are evaluating $R, Q$ and $P$ in the static limit, $q^{0}=0$. From Eq. (5.2) we then obtain

$$
\begin{aligned}
\pi_{00}(0, \vec{q}) & =\pi_{L}(0, \vec{q}) \\
\pi_{0 i}(0, \vec{q}) & =\pi_{i 0}(0, \vec{q})=0 .
\end{aligned}
$$

Eq. (5.1) then yields

$$
\Gamma_{0}(0, \vec{q} \rightarrow 0)=-\left(\frac{G_{F} \mathcal{A}}{\sqrt{2} e}\right) \gamma_{0}\left(1-\gamma_{5}\right) \pi_{L}(0, \vec{q} \rightarrow 0) .
$$

Using the definition of the Debye screening length,

$$
r_{D}^{-2}=\pi_{L}(0, \vec{q} \rightarrow 0),
$$

we finally obtain for the induced neutrino charge by the use of Eq. (2.13):

$$
e_{\text {ind }}^{(\nu)}=-\frac{G_{F} \mathcal{A}}{\sqrt{2} e r_{D}^{2}}(1-\lambda),
$$

where, as stated before, $\lambda$ is the helicity of the neutrino. Thus, it is clear that only the left-handed neutrinos have an induced charge. The induced charge for the right handed neutrinos vanish since they have no weak interactions. If they interact via some other weaker interaction, then of course they also acquire an induced charge, but the magnitude of that will be further suppressed. Also, note that $e_{\text {ind }}^{(\nu)} \propto e$, since $r_{D}^{2} \propto e^{-2}$ which follows from Eq. (5.15). 


\section{Generalization to massive neutrinos}

The generalization of our previous results to massive neutrinos is straightforward, although there are several important differences. The effective charge is defined by Eq. (2.12), but the expression for the spinor projection operator $S(k)$, as well as the expressison for $\Gamma_{0}$, differ from the previous case. The spinor projection operator $S(k)$ is given, for massive particles, by

$$
S(k)=u(k) \otimes \bar{u}(k)=\frac{1}{2}(\not k+m)\left(1+\lambda \gamma^{5} \phi\right),
$$

where $s^{\mu}$ is the spin polarization vector which, for helicity states, is given by

$$
s^{\mu}=\frac{1}{m}(|\vec{k}|, E \vec{k} /|\vec{k}|) .
$$

Therefore, although Eq. (2.12) remains valid for massive neutrinos, Eq. (2.13) does not. To proceed, we consider the cases of Dirac and Majorana neutrinos separately.

\subsection{Dirac case}

For Dirac neutrinos, $\Gamma_{\lambda}$ remains to be given by Eq. (4.12) and the effective charge by Eq. (2.12). The formula for the effective charge of Dirac neutrinos is now obtained by substituting Eq. (6.1) in Eq. (2.12), yielding

$$
e_{\text {ind }}^{\left(\nu^{D}\right)}=-\frac{G_{F} \mathcal{A}}{\sqrt{2} e r_{D}^{2}}\left(1-\frac{\lambda|\vec{k}|}{E}\right) .
$$

Specializing this formula to the case of massless neutrinos we recover Eq. (5.16), as it should be. In the opposite limit of non-relativistic neutrinos, we see that both helicity states have the same effective charge, which is equal to half the value of the charge of the left-handed neutrino in the massless case.

\subsection{Majorana case}

For (massive) Majorana neutrinos we again have to use the spinor projection operator appropriate for massive particles, given in Eq. (6.1). However, the formula for $\Gamma_{\lambda}$ is modified as follows. Going back to Eq. (4.9), it is important to recognize that each one of the neutrino field operators $\nu(y)$ and $\bar{\nu}(y)$ can be

contracted with either one of the same field operators that come from the factor $\mathcal{L}_{\text {int }}^{(\text {weak })}$. The reason is that for a Majorana neutrino the field operator is self-conjugate and therefore $\nu(y)$ can be contracted with not only $\bar{\nu}(y)$ but also with itself. The net result of adding these two possible contractions is that the expression for $\Gamma_{\lambda}$ given in Eq. (4.12) is replaced by [16]

$$
\Gamma_{\lambda}^{(M)}=-\frac{G_{F}}{\sqrt{2} e}\left(-2 \gamma^{\rho} \gamma^{5}\right)\left(\mathcal{A} \pi_{\lambda \rho}+\mathcal{B} \pi_{\lambda \rho}^{5}\right) .
$$

Substituting this formula and the projection operator given above for the massive case, into Eq. (2.12) we then obtain the effective charge for Majorana neutrinos

$$
e_{\text {ind }}^{\left(\nu^{M}\right)}=\frac{\sqrt{2} G_{F} \mathcal{A}}{e r_{D}^{2}} \frac{\lambda|\vec{k}|}{E} .
$$

We notice the following features: $(i)$ the positive and negative helicity states have opposite effective charge; (ii) in the masless limit the result for the negative helicity state is the same one obtained in the massless Dirac case and in the Weyl case, while the positive helicity state has the opposite value of the charge. This is not surprising since the right-handed component of the Majorana neutrino is just the CPT conjugate of the left-handed one. 


\section{Numerical estimates}

In order to obtain numerical estimates, we note that since $|\vec{k}| / E \leq 1$, the magnitude of the induced charge is maximum if the neutrino is massless. Thus, in this section, we use Eq. (5.16), which is valid for massless neutrinos. We see that we need to know two things in order to obtain a numerical estimate for the induced charge of the neutrino, viz., $\mathcal{A}$ and $r_{D}$. The first is easy, and is given by the standard model of electroweak interactions. In fact, one obtains

$$
\mathcal{A}= \begin{cases}2 \sin ^{2} \theta_{W}+\frac{1}{2} & \text { for } \nu_{e} \\ 2 \sin ^{2} \theta_{W}-\frac{1}{2} & \text { for } \nu_{\mu}, \nu_{\tau} .\end{cases}
$$

For massless $\nu_{e}$, our result exactly reproduces the results of Oraevsky and Semikoz [6]. Our formulas can be used to obtain the induced charges for the $\nu_{\mu}$ and $\nu_{\tau}$ as well, even if they are massive particles.

One curious thing to note is the fact that the induced charge of the $\nu_{e}$ is different from that of $\nu_{\mu}$ or $\nu_{\tau}$ since the $\nu_{e}$ 's interact with the electrons of the medium via both charge and neutral currents. Thus, if neutrinos mix, when they oscillate during their propagation through a medium, the induced charges also oscillate. Using $\sin ^{2} \theta_{W}=0.23$, we see that

$$
\frac{e_{\text {ind }}^{\left(\nu_{e}\right)}}{e_{\text {ind }}^{\left(\nu_{\mu}\right)}}=-24 .
$$

It therefore seems that this oscillation of charges should be a fantastic phenomenon, judging by the fact that the ratio of the induced charges is large, and also is negative. However, that is not the case because the magnitude of the induced charge appears to be extremely small. In fact, putting numbers in Eq. (5.16), one obtains

$$
e_{\text {ind }}^{\left(\nu_{e}\right)}=-2 \cdot 10^{-32} \times\left(\frac{1 \mathrm{~cm}}{r_{D}}\right)^{2} .
$$

To proceed, we need the value of $r_{D}$ for the background. This can be obtained either from the results of Ref. [8] or from standard texts on plasma physics. For a background consisting of non-relativistic electrons at temperature $T$, the Debye radius is given by

$$
r_{D}^{2}=\frac{T}{n_{e} e^{2}}
$$

where $n_{e}$ is the electron number densityf. In order for the induced neutrino charge to be detectable in experiments, the values of $T$ and $n_{e}$ must be such that the resulting Debye radius is small enough. While this is not the case for any known plasma, the methods presented here may prove useful in applications to similar situations where more exciting results may be obtained.

Note added in proof : After this work was submitted for publication, we were made aware of a paper by Altherr and Kainulainen [17] where the one-loop electromagnetic vertex of neutrinos has been calculated in a medium. The calculation agrees with that of Ref. [8]. These authors specifically noted that an induced charge appears in the medium. No effort was made to make contact with the Debye radius.

Acknowledgements : The work of PBP was supported by a grant from the Department of Energy.

\footnotetext{
${ }^{2}$ Formulas appearing in books on Plasma Physics usually have a factor $4 \pi$ in the denominator on the right hand side since their definition of electric charge is different.
} 


\section{References}

[1] For references and a recent review, see, e.g., P B Pal: Int. J. Mod. Phys. A7 (1992) 5387.

[2] S P Mikheyev, A Y Smirnov: Nuovo Cimento C9 (1986) 17.

[3] L Wolfenstein: Phys. Rev. D17 (1978) 2369.

[4] J F Nieves, P B Pal: Phys. Rev. D40 (1989) 1693.

[5] V B Semikoz, Y A Smorodinskii: JETP 68 (1989) 20.

[6] V N Oraevsky, V B Semikoz: Physica 142A (1987) 135.

[7] V N Oraevskii, A Y Plakhov, V B Semikoz, Y A Smorodinskii, JETP 66 (1987) 890.

[8] J C D'Olivo, J F Nieves, P B Pal: Phys. Rev. D40 (1989) 3679.

[9] J C D’Olivo, J F Nieves, P B Pal: Phys. Rev. Lett. 64 (1990) 1088.

[10] C Giunti, C W Kim, W P Lam: Phys. Rev. D43 (1991) 164.

[11] R F Sawyer: Phys. Rev. D46 (1992) 1180.

[12] C Giunti, C W Kim, U W Lee, W P Lam: Phys. Rev. D45 (1992) 1557.

[13] J B Adams, M A Ruderman, C-H Woo: Phys. Rev. 129 (1963) 1383.

[14] E Braaten, D Segel: "Neutrino energy loss from plasma process at all temperatures and densities", Northwestern University preprint NUHEP-TH-93-1 (January 1993).

[15] J F Nieves, P B Pal: Phys. Rev. D39 (1989) 652, 40 (1989) 2148(E).

[16] For details of this procedure, see e.g., R N Mohapatra and P B Pal: Massive neutrinos in Physics and Astrophysics (World Scientific, Singapore 1991), Sec. 10.1.2.

[17] T. Altherr and K. Kainulainen: Phys. Lett. B262 (1991) 79. 

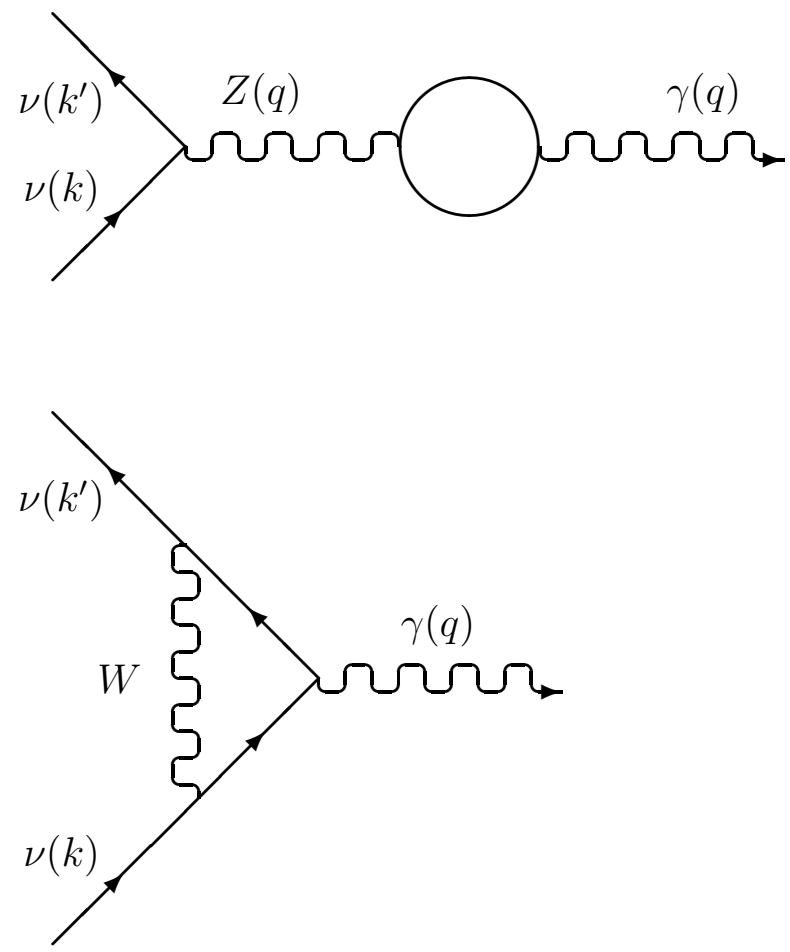

(b)

Figure 1: 1-loop diagrams for the effective electromagnetic vertex of neutrinos which contribute in the limit that the 4-momenta of the $W$ and the $Z$ lines are neglected.

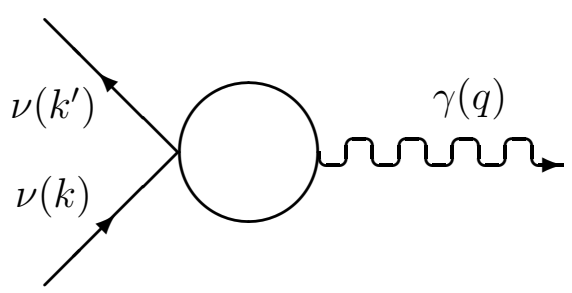

Figure 2: The diagrams of Fig. 11 in the limit of infinitely heavy $W$ and $Z$ masses.

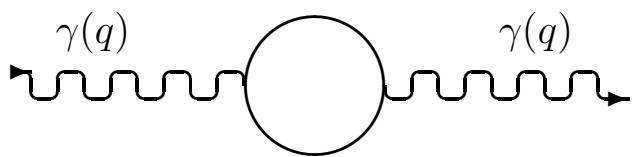

Figure 3: 1-loop diagram for the vacuum polarization of the photon. 